\title{
Extraordinary transmission through a silver film perforated with cross shaped hole arrays in a square lattice
}

\author{
Chia-Yi Chen, Ming-Wei Tsai, Tzu-Hung Chuang, Yi-Tsung Chang, and Si-Chen Lee ${ }^{\text {a) }}$ \\ Department of Electrical Engineering, Graduate Institute of Electronics Engineering, National Taiwan \\ University, Taipei, 10617 Taiwan, Republic of China
}

(Received 13 April 2007; accepted 8 July 2007; published online 7 August 2007)

\begin{abstract}
In this work, the transmission through cross shaped hole with different sizes but the same lattice constant was measured with the polarized light to investigate the variation of localized charge oscillation around the hole. The comparison between the transmission intensities through the similarly arranged periodic hole array with different hole shapes, i.e., cross, square, and rectangular, were also measured. The cross shaped hole gives rise to a larger transmission of light than those perforated with square or rectangular hole. (C) 2007 American Institute of Physics.
\end{abstract}

[DOI: $10.1063 / 1.2767183$ ]

Extraordinary transmission through an optically thick metal film perforated with subwavelength hole arrays is discovered by Ulrich. ${ }^{1}$ It is widely accepted that the surface plasmon resonance on the metal film is the main mechanism responsible for light enhancement. ${ }^{2}$ When the light is incident on the subwavelength hole arrays, the extra momentum provided by the arrays could couple the incident light with the surface plasmon on the metal film and eventually convert to radiation. ${ }^{2,3}$ The dependence of the transmission spectra on the metal, ${ }^{4,5}$ hole size, ${ }^{6,7}$ hole depth, ${ }^{8}$ hole shape, ${ }^{9-15}$ and the polarization of incident light has been demonstrated theoretically and experimentally. ${ }^{4-15}$ When the size of the hole exceeds half of the lattice constant, a splitting of the transmission spectra is observed. ${ }^{6}$ The influence of the hole shape is due to the effect of localized surface plasmon (LSP) around the aperture. ${ }^{13-15}$ When light impinges on a rectangular hole arrays, the coupling between the LSPs on the long edges of rectangular hole cause the cutoff wavelength to increase. ${ }^{14}$ As the aspect ratio increases large enough, this coupling effect becomes so pronounced that the charge dipole oscillations on the short edges disappear. ${ }^{9}$ To discuss the effect of hole shape, Ulrich ${ }^{16}$ and Moller and co-workers ${ }^{17-20}$ have studied the transmission through cross shaped metal meshes in infrared region by transmission line theory.

In this work, the transmissions of radiation through cross shaped hole with different sizes but the same lattice constant were measured with the polarized light. The transmission spectra of the square and rectangular holes arranged in a similar square lattice were also measured to study the influence of hole shape on the localized charge oscillations.

After the photoresist was spun on the silicon wafer and pattern transfer, 100 -nm-thick Ag metal films were thermally deposited and lifted off to form cross shaped hole arrays. The cross shaped holes are symmetric to the $X$ axis and $Y$ axis and arranged in a square lattice with lattice constant $a$ $=10 \mu \mathrm{m}$. Parameters of cross shaped hole and top view of the structure are defined and shown in Fig. 1. A Bruker IFS $66 \mathrm{v} / \mathrm{s}$ system was used to measure transmission spectrum. Polarized light is normally incident on the $\mathrm{Ag}$ side of the samples. The wave number resolution of the measurement was $8 \mathrm{~cm}^{-1}$.

\footnotetext{
${ }^{a)}$ Electronic mail: sclee@cc.ee.ntu.edu.tw
}

Figures 2(a)-2(c) display the zero-order transmission spectra of the cross shaped hole arrays with $W=3 \mu \mathrm{m}, L 1$ $=7 \mu \mathrm{m}$, and $L 2$ varying from 7, 5 to $3 \mu \mathrm{m}$, respectively. The lattice constants $a$ are all $10 \mu \mathrm{m}$ for these samples. Measurements with unpolarized, $X$-polarized, and $Y$-polarized lights are denoted by black, red, and blue curves, respectively. In Figs. 2(a)-2(c), the major peaks at $35.7 \mu \mathrm{m}$ are the degenerate $(0, \pm 1)$ and $( \pm 1,0) \mathrm{Ag} / \mathrm{Si}$ modes. In Fig. 2(a), where $L 2=7 \mu \mathrm{m}$, the intensities of the peaks in $X$-polarized and $Y$-polarized transmissions are almost the same. When $L 2$ decreases from 7 to $5 \mu \mathrm{m}$, as shown in Fig. 2(b), the intensity of the degenerate $( \pm 1,0) \mathrm{Ag} / \mathrm{Si}$ modes in $X$-polarized transmission decreases substantially, and no peak splitting is observed in $(0, \pm 1) \mathrm{Ag} / \mathrm{Si}$ modes in $Y$-polarized transmission even though $L 2=5 \mu \mathrm{m}$ cross which is equal to the half of the lattice constant. When $L 2=3 \mu \mathrm{m}$, as shown in Fig. 2(c), the hole becomes rectangular shape, the whole transmission is dominated by $Y$-polarized light and that under $X$-polarized light almost disappears completely. This is because the strong coupling of localized surface plasmon to the long edge of the rectangular holes results in the disappearance of the $( \pm 1,0) \mathrm{Ag} / \mathrm{Si}$ mode in $X$-polarized light. ${ }^{9}$

Figures 2(d)-2(f) display the zero-order transmission spectra of the cross shaped hole array with $W=5 \mu \mathrm{m}, L 1$ $=7 \mu \mathrm{m}$, and $L 2$ ranging from 7, 6 to $5 \mu \mathrm{m}$. It can be seen clearly in Fig. 2(d) where $L 2=7 \mu \mathrm{m}$ that the degenerate $(0, \pm 1)$ and $( \pm 1,0) \mathrm{Ag} / \mathrm{Si}$ modes split into two peaks at 34.7 and $36.3 \mu \mathrm{m}$ under unpolarized light. As $L 2$ decreases from 7 to $6 \mu \mathrm{m}$, the transmission intensity of the peak decreases and the mode splitting is not apparent under $X$-polarized light, but the split still can be seen under $Y$-polarized light, as shown in Fig. 2(e). At $L 2=5 \mu \mathrm{m}$, it becomes a rectangular shape hole, the transmission intensity at $36.3 \mu \mathrm{m}$ under

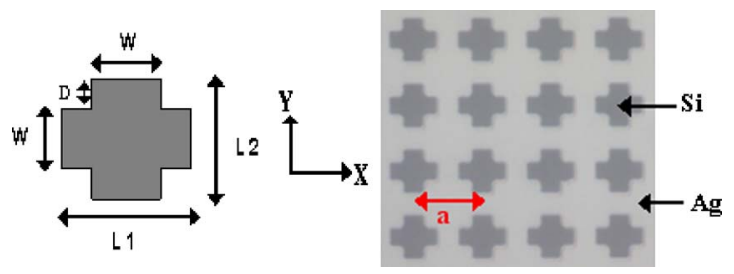

FIG. 1. (Color online) Definition of the parameters of the cross shaped hole and the top view of the sample. Lattice constant $a$ is $10 \mu \mathrm{m}$. 

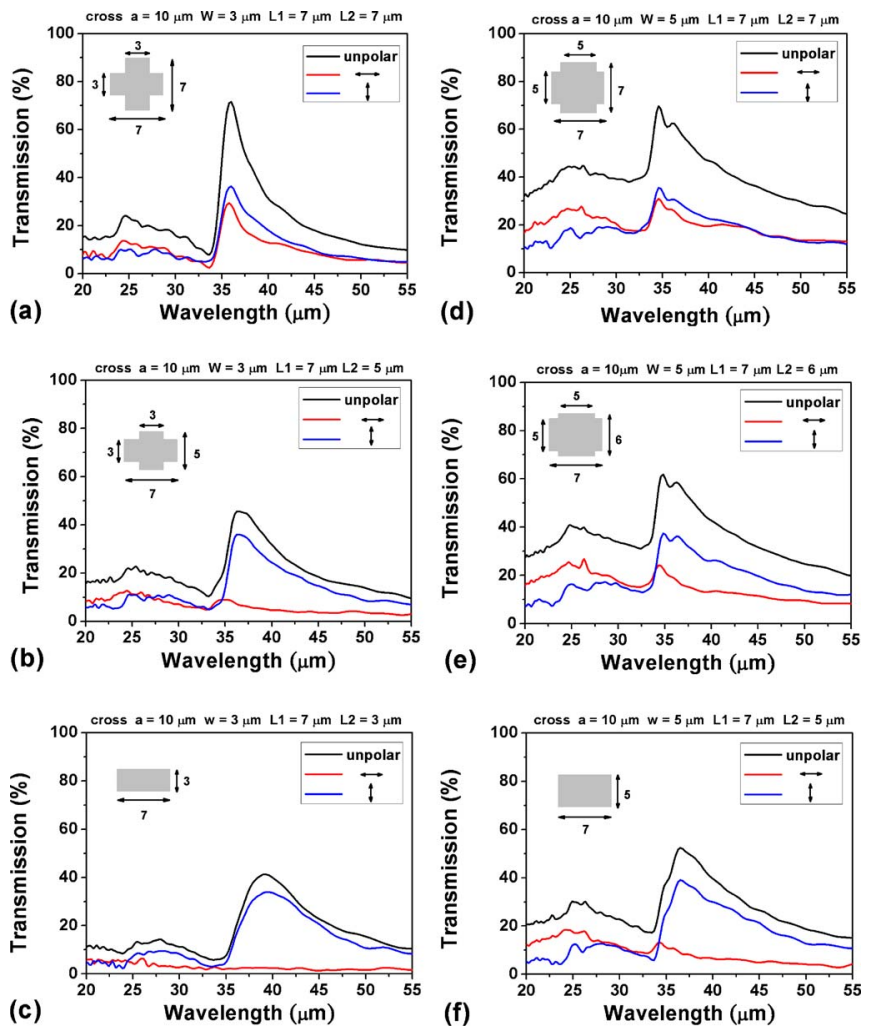

FIG. 2. (Color online) Zero-order transmission spectra of cross shaped hole arrays with $W=3 \mu \mathrm{m}, L 1=7 \mu \mathrm{m}$, and $L 2=$ (a) 7, (b) 5, and (c) $3 \mu \mathrm{m}$, whereas $W=5 \mu \mathrm{m}, L 1=7 \mu \mathrm{m}$, and $L 2=$ (d) 7 , (e) 6 , and (f) $5 \mu \mathrm{m}$ at normal incidence with unpolarized, $X$-polarized, and $Y$-polarized lights; the lattice constant $a$ is $10 \mu \mathrm{m}$.

$X$-polarized light is almost zero and the intensity of the peak at $34.7 \mu \mathrm{m}$ also decreases substantially. However, the double peak characteristic can still be seen. When $L 2$ decreases, the charge dipole oscillations along the $X$ direction become harder to excite. Then the transmission of $X$-polarized light decreases rapidly and whole spectrum is dominated by the transmission of $Y$-polarized light eventually.

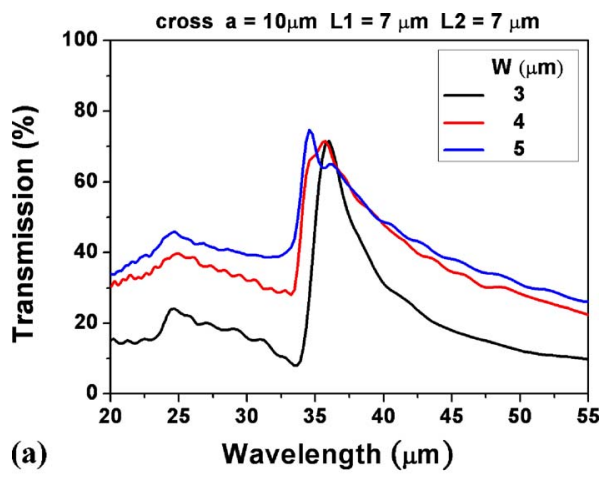

(a)

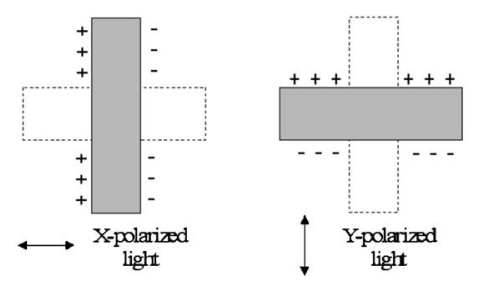

FIG. 3. (Color online) Zero-order transmission spectrum of cross shaped hole arrays with $L 1=L 2=7 \mu \mathrm{m}$ and $W=3,4$, and $5 \mu \mathrm{m}$. The lattice constant $a$ is $10 \mu \mathrm{m}$. (b) Schematic illustration of localized charge oscillations around the cross shaped hole with respect to $X$-polarized light and $Y$-polarized light. $Y$-polarized light.
Downloaded 04 Mar 2009 to 140.112 .113 .225 . Redistribution subject to AIP license or cop $10 \mu \mathrm{m}$.
Figure 3(a) shows transmission spectrum of cross shaped hole arranged in square lattice with the same $L 1$ and $L 2$ $(7 \mu \mathrm{m})$ but different $W$ from 3,4 to $5 \mu \mathrm{m}$. As the $W$ becomes larger, the peak of $\mathrm{Ag} / \mathrm{Si}$ mode splits off. According to Tsai et al., ${ }^{6}$ the transmission peak of surface plasmon mode peak splits when hole size is larger than the half of lattice constant because of the Bragg reflection of plasmon polariton resulting in two standing waves. Thus, it can be concluded that when $W$ of cross is close to half of the lattice constant, the charge dipole oscillations on the $D$ edges (defined in Fig. 1), as shown in Fig. 3(b), are similar to a rectangular hole with length close to the half of the lattice constant. Thus the split of peak can be seen clearly.

In order to understand the characteristics of cross shaped hole arrays further, the transmission spectra of hole arrays with different hole shapes but almost the same area are also measured, as shown in Fig. 4. The lattice constant $a$ is $10 \mu \mathrm{m} ; L 1$ and $L 2$ of the cross are the same and denoted by $L$. $W$ is defined the same as before. $W$ and $L$ of the rectangular hole are the width and length, respectively. $L$ of square hole is the side length. It is found that as the $W / L$ ratio of the cross hole becomes smaller, the full width at half maximum (FWHM) of the transmission peak broaden, and the position of the peak shifts from 35.1 to $40.3 \mu \mathrm{m}$. The redshift of the resonance peak can also be seen when the square hole transformed to rectangular hole gradually. It is because of the increase of the cutoff wavelength due to the strong coupling of the localized surface plasmon on the long edges of rectangular hole. ${ }^{14}$ Therefore, these two similar redshifts provide another evidence that for small $\mathrm{W}$, charge dipole oscillations
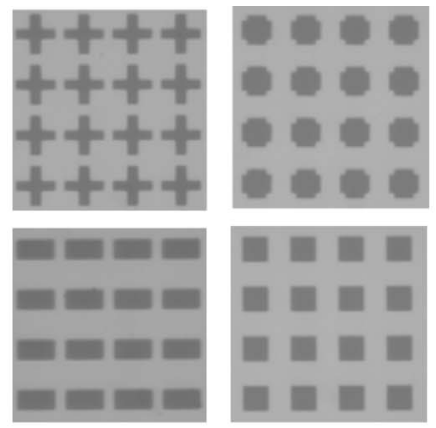

(a)

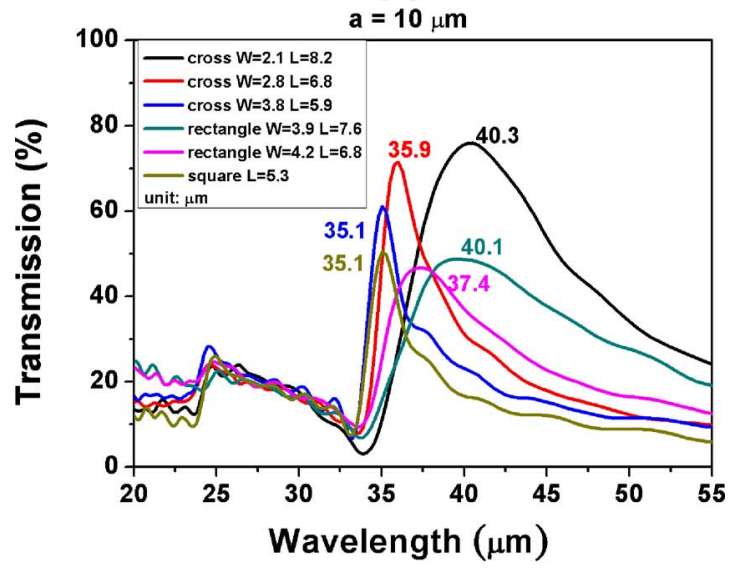

(b)

FIG. 4. (Color online) (a) Top views of cross shaped hole, rectangular hole, and square hole arrays. (b) Zero-order transmission spectra of the cross shaped, rectangular, and square hole arrays with different $W$ and $L$. Lattice 

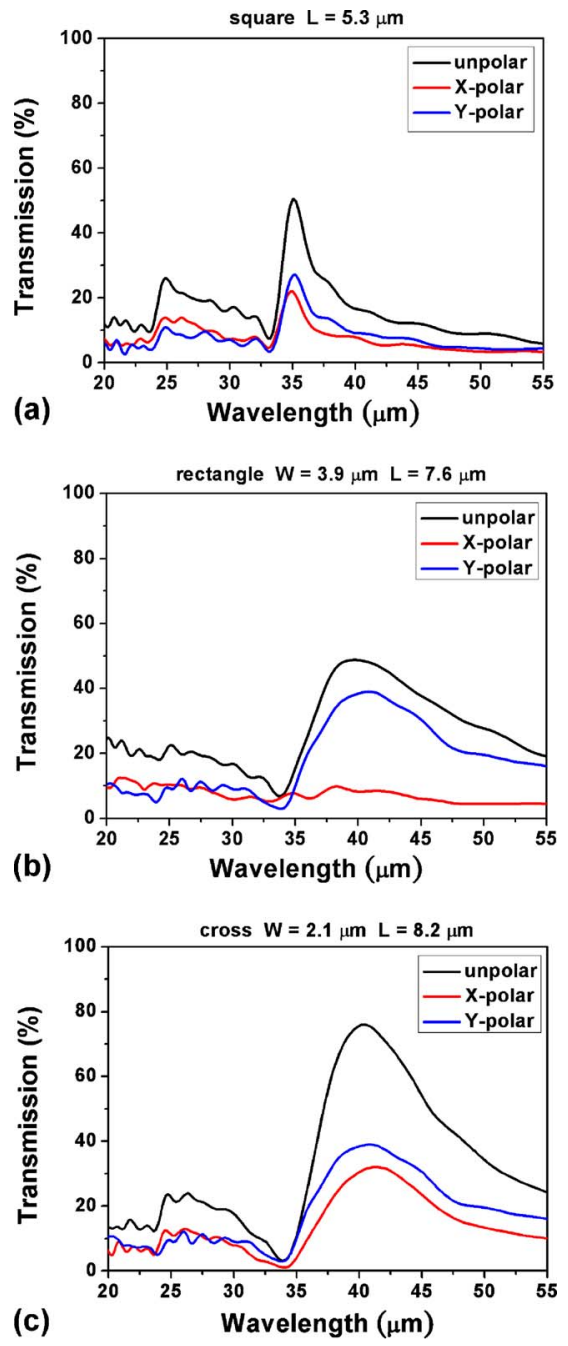

FIG. 5. (Color online) Zero-order transmission spectra of (a) square, (b) rectangular, and (c) cross shaped hole arrays under unpolarized, $X$-polarized and $Y$-polarized lights.

of cross shaped hole are really rectangularlike. The increase of FWHM is caused by the effect of radiation damping caused by the scattering of plasmon with the apertures on metal film. ${ }^{21,22}$ This radiation damping effect would be enlarged as the aperture size increase because of more strong scattering between plasmon and apertures.

Another interesting result is also observed. The transmission peak intensity of cross shaped hole arrays with different sizes is all larger than those of rectangular and square hole arrays, even though the hole areas are almost the same. When the polarized light is incident on the square hole arrays, the transmission intensity of $X$-polarized and $Y$-polarized lights are almost the same, as shown in Fig. 5(a), because the charge dipole oscillations induced by polarized light are symmetric around the hole. For rectangular hole arrays, the whole transmission is dominated by $Y$-polarized light. The transmission of $X$-polarized light is weak, as shown in Fig. 5(b). Comparing Figs. 5(a) and 5(b), it is found that the coupling efficiency of $Y$-polarized light to rectangular hole arrays is larger than that of square hole arrays. The transmission spectra of narrow cross hole arrays with different polarized lights are shown in Fig. 5(c). It is clear that the transmission peak amplitude of $X$-polarized and $Y$-polarized lights are all larger than those of square hole arrays, but similar to those of rectangle hole with $Y$-polarized light. This indicates that the cross shaped hole arrays could be regarded as the combination of two rectangular hole arrays where the long edges are perpendicular to the polarization directions $X$ and $Y$. As a result, the coupling efficiency of cross shaped hole arrays to unpolarized light $(\sim 77 \%)$ is much larger than both square and rectangle hole arrays $(\sim 50 \%)$ even though they are all symmetric hole shape with almost the same area.

In conclusion, it is found that when the $W / L$ ratio is small, the charge dipole oscillations around cross shaped hole are just like those of a rectangular hole when the polarized light with the polarization perpendicular to the long edge of rectangle. Besides, the cross shaped hole arrays exhibit more efficient coupling of incident light than both square and rectangular hole arrays with the same area and lattice constant.

This work is supported by the National Science Council of Republic of China under Contract No. NSC 95-2120-M002-007.

${ }^{1}$ R. Ulrich, Proceedings of the Optical and Acoustic Micro-Electronic Symposium, New York, 16-18 April 1974 (unpublished).

${ }^{2}$ H. F. Ghaemi, Tineke Thio, D. E. Grupp, T. W. Ebbesen, and H. J. Lezec, Phys. Rev. B 58, 6779 (1998).

${ }^{3}$ T. W. Ebbesen, H. J. Lezec, H. F. Ghaemi, T. Thio, and P. A. Wolff, Nature (London) 391, 667 (1998).

${ }^{4}$ Tineke Thio, H. F. Ghaemi, H. J. Lezec, P. A. Wolff, and T. W. Ebbesen, J. Opt. Soc. Am. B 16, 1743 (1999).

${ }^{5}$ F. Przybilla, A. Degiron, J.-Y. Laluet, C. Genet, and T. W. Ebbesen, J. Opt. A, Pure Appl. Opt. 8, 458 (2006).

${ }^{6}$ Ming-Wei Tsai, Tzu-Hung Chuang, Hsu-Yu Chang, and Si-Chen Lee, Appl. Phys. Lett. 88, 213112 (2006).

${ }^{7}$ Shaun M. Williams, Amanda D. Stafford, Trisha M. Rogers, Sarah R. Bishop, and James V. Coe, Appl. Phys. Lett. 85, 1472 (2004).

${ }^{8}$ A. Degiron, H. J. Lezec, W. L. Barnes, and T. W. Ebbesen, Appl. Phys. Lett. 81, 4327 (2002).

${ }^{9}$ Ming-Wei Tsai, Tzu-Hung Chuang, Hsu-Yu Chang, and Si-Chen Lee, Appl. Phys. Lett. 89, 093102 (2006).

${ }^{10}$ K. L. van der Molen, F. B. Segerink, N. F. Van Hulst, and L. Kuipers, Appl. Phys. Lett. 85, 4316 (2004).

${ }^{11}$ K. J. Klein Koerkamp, S. Enoch, F. B. Segerink, N. F. van Hulst, and L. Kuipers, Phys. Rev. Lett. 92, 183901 (2004).

${ }^{12}$ R. Gordon, A. G. Brolo, A. Mckinnon, A. Rajora, B. Leathem, and K. L. Kavanagh, Phys. Rev. Lett. 92, 037401 (2004).

${ }^{13}$ A. Degiron, H. J. Lezec, N. Yamamoto, T. W. Ebbesen, Opt. Commun. 239, 61 (2004).

${ }^{14}$ Reuven Gordon and Alexandre G. Brolo, Opt. Express 13, 1933 (2005).

${ }^{15}$ A. Degiron and T. W. Ebbesen, J. Opt. A, Pure Appl. Opt. 7, S90 (2005).

${ }^{16}$ R. Ulrich, Appl. Opt. 7, 1987 (1968).

${ }^{17}$ V. P. Tomaselli, D. C. Edewaard, P. Gillan, and K. D. Moller, Appl. Opt. 20, 1361 (1981).

${ }^{18}$ K. D. Moller, J. B. Warren, J. B. Heaney, and C. Kotecki, Appl. Opt. 35, 6210 (1996).

${ }^{19}$ Karl D. Moller, Oren Sternberg, Haim Grebel, and Kenneth P. Stewart, Appl. Opt. 41, 1942 (2002).

${ }^{20}$ K. D. Moller, O. Sternberg, H. Grebel, and Philippe Lalanne, J. Appl. Phys. 91, 9461 (2002).

${ }^{21}$ D. S. Kim, S. C. Hohng, V. Malyarchuk, Y. C. Yoon, Y. H. Ahn, K. J. Yee, J. W. Park, J. Kim, Q. H. Park, and C. Lienau, Phys. Rev. Lett. 91, 143901 (2003).

${ }^{22}$ Roland Muller, Viktor Malyarchuk, and Christoph Lienau, Phys. Rev. B 68, 205415 (2003). 